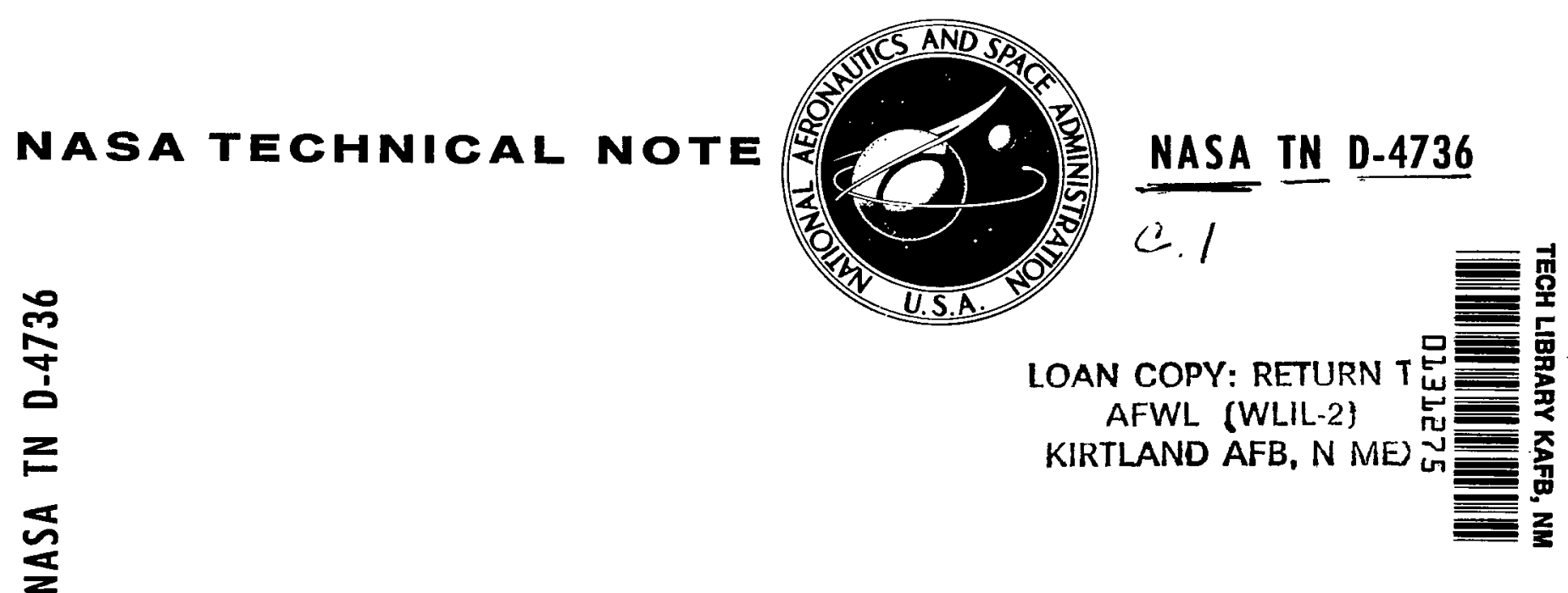

\title{
SHAPE OF A
}

\section{MAGNETICALLY BALANCED ARC}

by Marvin E. Goldstein and Willis H. Braun

Lewis Research Center

Cleveland, Obio

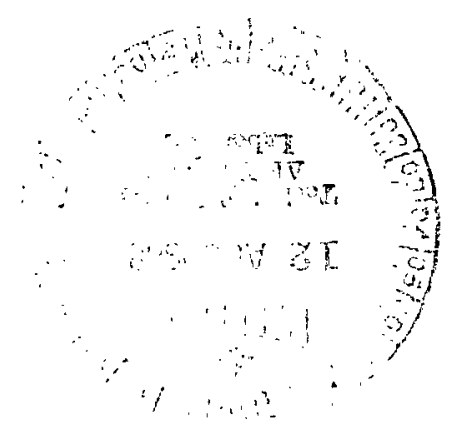

NatiOnal aeronauticS AND SPACE ADMINISTRATION - WASHINGTON, D. C. • AUGUST 1968 
SHAPE OF A MAGNETICALLY BALANCED ARC

By Marvin E. Goldstein and Willis H. Braun

Lewis Research Center

Cleveland, Ohio

\section{NATIONAL AERONAUTICS AND SPACE ADMINISTRATION}

For sole by the Clearinghouse for Federal Scientific and Technical Information Springfield, Virginio 22151 - CFSTI price $\$ 3.00$ 


\section{ABSTRACT}

An improved model is developed for predicting the shape of an electric arc balanced in transverse convective and magnetic fields. The improvement over the previous model is in the more accurate description of the external flow field. The techniques used are based on conformal mapping and are an extension of those methods developed for problems of free surface flows. 


\title{
SHAPE OF A MAGNETICALLY BALANCED ARC \\ by Marvin E. Goldstein and Willis H. Braun \\ Lewis Research Center
}

\author{
SUMMARY
}

An improved model is developed for predicting the shape of an electric arc balanced in transverse convective and magnetic fields. The improvement over the previous model is in the more accurate description of the external flow field. The techniques used are based on conformal mapping and are an extension of those methods developed for problems of free surface flows.

\section{INTRODUCTION}

The importance of electric arcs interacting with transverse convective and magnetic fields has greatly increased during the last decade because of numerous applications of electric arc devices to aerospace technology (ref. 1). The two types of configurations that have been used to study this threefold interaction are (1) the so-called rail accelerator in which the arc moves through a quiescent fluid and (2) the magnetically balanced arc that is discussed herein (see fig. 1). In this type, a magnetic field perpendicular to both a channel flow and an electric field is adjusted so that the electromagnetic force $\vec{j} \times \vec{B}$ acting on the arc just balances the hydrodynamic forces. The experiments show that this type of arc tends to behave much like a cylindrical solid body (ref. 1).

A simple model, from which the cross-sectional shape of the arc could be determined, was proposed in reference 2. That paper pointed out that some of the features of the model were expected to be inaccurate in detail but that the type of analysis employed was expected to be correct. In the present report, the arc model described in reference 2 is improved by eliminating some of the inaccuracies concerned with the external flow about the arc, although the same model for the internal structure of the arc is retained.

Those features of the model presented in reference 2 that are used herein are the assumptions that the hot fluid recirculates within the arc column while the cold external fluid flows around the column, much as it would flow about a solid body; and, further- 


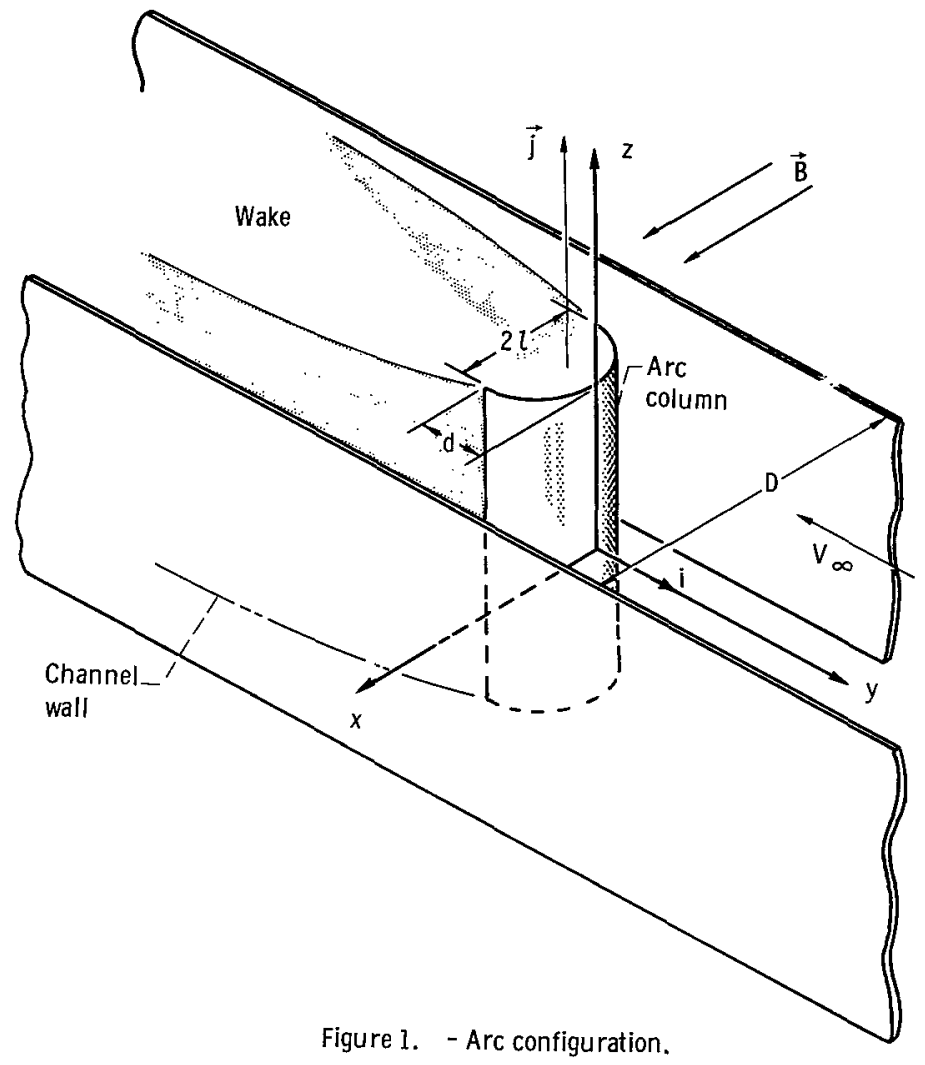

more, the arc can be considered two-dimensional (all variations occur in the $\mathrm{x}, \mathrm{y}$-plane shown in fig. 1); the current density $j$ is uniform across the arc cross section, and Hall currents can be neglected. Under these conditions, the magnetic force per unit volume is constant and equal to $\hat{\mathrm{ijB}}$, where $\hat{\mathrm{i}}$ is the unit vector in the direction opposite to the upstream velocity (as shown in fig. 1). Now $\hat{\mathrm{ij} B}$ is a conservative force and, hence, can be balanced by a pressure gradient. Reference 2 also assumed that both inertial and viscous forces could be neglected within the arc. With these approximations, the momentum equation for the hot fluid within the arc is

$$
\nabla p_{i}=\hat{i j B}
$$

where $p_{i}$ is the pressure within the arc. The external flow was taken to be inviscid and incompressible. (Most experiments were carried out at sufficiently low velocities so that compressibility effects were not important). The purpose of the present analysis is to find the shape of the arc that will make the external potential flow compatible with the solutions to equation (1) within the arc. (The matching conditions are discussed in the next section.) At this point, it is desirable to deviate from the analysis given in reference 2. 
The model for the potential flow given in reference 2, although it satisfied the appropriate matching conditions along the surface of the arc, had several serious drawbacks. First, the arc turned out to be a semi-infinite body that had to be artificially truncated. Second, the streamlines gradually converged at large distances from the arc instead of becoming parallel. Third, the model did not take into account the wake region behind the arc. Fourth, the model differed from the experimental situation in that it did not take into account the presence of channel walls that could have a large effect on the wake region, even if the walls are quite far apart. In the present model, all these drawbacks are eliminated.

\section{SYMBOLS}
A cross-sectional area of arc
$\mathrm{B} \quad|\overrightarrow{\mathrm{B}}|$
$\vec{B} \quad$ magnetic flux density vector
D channel width
d longest dimension of arc in direction parallel to flow
$\mathrm{h}$ distance between stagnation point and point at upstream infinity in t-plane
I total current in arc
$\hat{\mathbf{i}} \quad$ unit vector in direction opposite to upstream velocity
j $\quad|\vec{j}|$
$\vec{j} \quad$ arc current density vector
$l \quad$ half the longest dimension of arc in direction perpendicular to flow
p pressure in external flow
$\mathbf{p}_{\mathbf{i}} \quad$ pressure in arc
$p_{0} \quad$ stagnation pressure
s $\quad \operatorname{Re}(t)$
$\mathbf{s}^{\dagger}$ distance from stagnation point measured along boundary streamline of arc
T intermediate complex variable
$\mathbf{T}_{\mathbf{I}}$ complex coordinate of point $\mathbf{I}$ in $\mathrm{T}$-plane
t intermediate complex variable
$u \quad x$-component of velocity of external flow 
V magnitude of velocity of external flow, $|\zeta|$

$V_{0}$ magnitude of velocity of external flow on boundary streamline of wake

$v_{s}$ magnitude of velocity of external flow on boundary streamline of arc, $|\zeta(s)|$

$\mathrm{V}_{\infty}$ magnitude of velocity far upstream of arc, $|\zeta(\mathrm{ih})|$

$\mathbf{v} \quad \mathrm{y}$-component of velocity of external flow

W complex potential, $\Phi+\mathbf{i} \Psi$

$\mathbf{x}$ coordinate perpendicular to flow and parallel to magnetic field

$x_{f} \quad x$-coordinate of boundary streamline of wake

$\mathbf{x}_{\mathbf{S}} \quad \mathbf{x}$-coordinate of boundary streamline of arc

y coordinate in direction opposite to flow

$\mathbf{y}_{\mathbf{f}} \quad \mathrm{y}$-coordinate of boundary streamline of wake

$\mathbf{y}_{\mathbf{s}} \quad \mathrm{y}$-coordinate of boundary streamline of arc

z complex variable, $\mathbf{x}+$ iy

$z_{f} \quad x_{f}+i y_{f}$

$z_{\mathbf{s}} \quad \mathbf{x}_{\mathbf{s}}+\mathrm{iy}_{\mathbf{s}}$

$\beta \quad$ included angle between two tangents to arc surface at stagnation point divided by $\pi$

$\Gamma \quad$ interior of first quadrant of $t$-plane

$\epsilon \quad$ convergence factor

$\zeta \quad$ complex conjugate velocity, $u-i v=d w / d z$

$\eta \quad\left(\frac{1+i \sqrt{t^{2}-1}}{t}\right)^{\beta} e^{i(1-\beta) \pi / 2}$

$\theta \quad \operatorname{Re}(\Omega)$

$\lambda \quad$ positive real number

$\rho$ density of external flow

$\tau \quad \operatorname{Im}(\Omega)$

$\Phi \quad$ velocity potential

$\varphi \quad$ angle between tangent to surface of arc and negative $\mathrm{y}$-axis

$\Psi \quad$ stream function

$\Psi_{0} \quad V_{\infty} D / 2 \pi h^{2}$ 
$\Omega \quad \mathrm{i} \ln \left(\zeta / \mathrm{V}_{\mathrm{o}} \eta\right)$

$\sim \quad$ asymptotically proportional to

Superscripts:

- $\operatorname{arc}$

* complex conjugate

\section{ANALYSIS}

\section{Boundary Conditions on Velocity}

Under the assumptions just discussed and from the integration of equation (1), the pressure $p_{i}$ at any point with coordinates $x, y$ (fig. 1) lying within the arc is given by

$$
p_{i}-p_{o}=j B y
$$

that is, the pressure in the arc is independent of $x$. This varying pressure in the arc must be balanced at the surface of the arc by the pressure field created by the external flow, and the arc surface must correspond to a streamline of the external flow. The distance measured along this bounding streamline (the arcs $\overparen{\mathrm{BA}}$ or $\overparen{\mathrm{BC}}$ in fig. 2) is denoted by $\mathrm{s}^{\dagger}$ and the magnitude of the velocity of the external flow along this streamline by $\mathrm{V}_{\mathrm{s}}$. Since the external flow was assumed to be incompressible and inviscid, Euler's equation along this streamline becomes

$$
-\rho \mathbf{V}_{\mathbf{s}} \frac{\partial \mathbf{V}_{\mathbf{s}}}{\partial \mathbf{s}^{\dagger}}=\frac{\partial \mathbf{p}}{\partial \mathbf{s}^{\dagger}}
$$

Now, since the internal and external pressure must balance along this bounding streamline,

$$
p=p_{i}(y) \quad \text { on } \overparen{B C}
$$



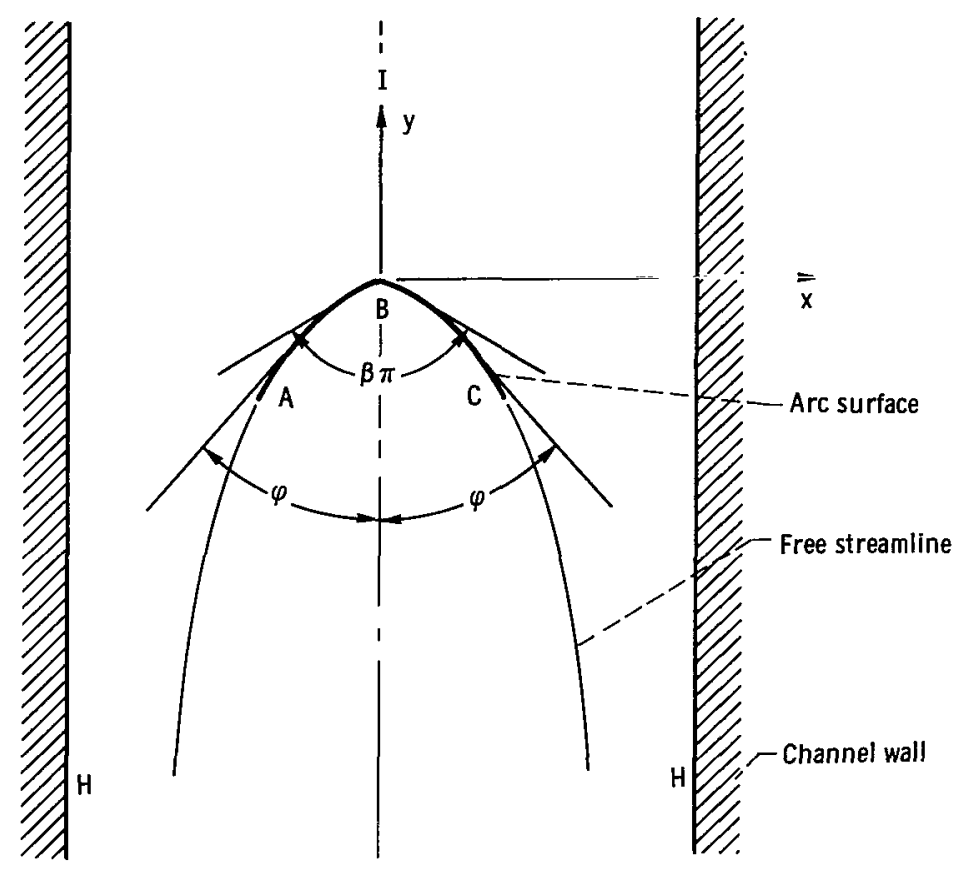

Figure 2. - Z-plane.

so that using equation (2) results in

$$
\begin{aligned}
\frac{\partial p}{\partial \mathbf{s}^{\dagger}}=\frac{\partial p_{\mathbf{i}}}{\partial \mathbf{s}^{\dagger}} & =\frac{d p_{i}}{d y} \frac{\partial y}{\partial s^{\dagger}} \\
& =j B \frac{\partial y}{\partial s^{\dagger}}=-j B \cos \varphi
\end{aligned}
$$

where $\varphi$ is the angle between the tangent to the boundary streamline and the negative $y$-axis as shown in figure 2. Substituting equation (5) into equation (3) yields

$$
\rho V_{s} d V_{s}=j B \cos \varphi d s^{\dagger}
$$

where $d V_{s}$ is understood to be the change in $V_{s}$ along $s^{\dagger}$.

Behind the arc is a wake. The temperature in the arc is very high. The fluid temperature in the wake is also high, but is somewhat cooler than that in the arc. Since the electrical conductivity of the fluids of interest for arc experiments (argon, air, etc.) drops rapidly to zero at a certain temperature and since the temperature must eventually drop off behind the arc, there will be a line on one side of which the fluid will be conducting and on the other side of which it will be nonconducting. This line is defined as the 
front boundary of the wake. Its location will be determined by the calculation; it is only necessary to assume that such a line exists. Thus the fluid in the wake is nonconducting so that the pressure acting on it is caused by only hydrodynamic forces. But this fluid is very hot (ref. 1), and therefore its density and the inertial forces are very low. Hence, to a very good approximation, the pressure is constant in the wake. The bounding streamlines of this wake must begin at the end points of the arc boundary $C$ and $A$ (i. e., they must be continuations of the bounding streamlines of the arc) and extend to $-\infty$, as shown in figure 2. Along these streamlines, the pressure on both sides must balance so that the pressure in the external flow is a constant along the streamlines $\overparen{\mathrm{CH}}$ and $\overparen{\mathrm{AH}}$ bounding the wake. Therefore, from Euler's equation, the magnitude of the velocity of the external flow is constant along these streamlines. This constant velocity is denoted by $V_{o}$, and the arc is assumed to be situated in a channel whose walls are separated by the distance D. In the final formulas, taking the limit $D-\infty$ will include the case where the arc is situated in an unbounded parallel flow. The boundary condition for the velocity along the channel walls is determined from the fact that it must be in the -y-direction, as must the velocity along the stagnation streamline $\overparen{\mathrm{IB}}$ (c.f., fig. 2 ).

\section{Boundary Conditions on Stream Function}

In order to completely determine the problem, it is sufficient to specify the values of the stream function $\Psi$ along the boundaries of the flow or equivalently along the boundaries of half the flow, since it is symmetric with respect to the y-axis. To specify these values requires that the bounding streamline of the arc $\overparen{\mathrm{BC}}$ be a continuation of the stagnation streamline $\overparen{\mathrm{IB}}$ and that the bounding streamline of the wake $\overparen{\mathrm{CH}}$ be a continuation of the arc $\overparen{\mathrm{BC}}$. Thus, $\Psi=0$ on $\overparen{\mathrm{IB}}, \overparen{\mathrm{BC}}$, and $\overparen{\mathrm{CH}}$. In addition, the channel wall corresponds to a streamline, or $\Psi$ is constant along the channel wall $\overparen{\mathrm{HH}}$.

\section{Calculation of Stream Function}

The problem is now completely specified, and its solution is obtained by conformally mapping the complex potential $\mathrm{W}$ defined by

$$
\mathrm{W}=\Phi+\mathrm{i} \Psi
$$

where $\Phi$ is the velocity potential, and the complex conjugate velocity $\zeta$ which is defined by

$$
\zeta=\frac{d W}{d z}=u-i v
$$

where $z=x+i y$, and $u$ and $v$ are the $x$ - and $y$-components of the velocity, respec- 
tively, into the upper half of an intermediate $t$-plane to be specified next.

Once $W$ and $\zeta$ are known as a function of the complex variable $t, z$ can be computed as a function of $t$ from

$$
z=\int \frac{d z}{d W} \frac{d W}{d t} d t=\int \zeta^{-1} \frac{d W}{d t} d t
$$

Thus, at least in principle, if $t$ is eliminated, $W$ and $\zeta$ can be found as functions of $z$. This yields the solution of the problem.

First consider the $\mathrm{W}$-plane. From the previous discussion, the arcs $\overparen{\mathrm{IB}}, \overparen{\mathrm{BC}}$, and $\widehat{\mathrm{CH}}$ must lie along the line $\Psi=0$, and the wall streamline $\widehat{\mathrm{IH}}$ must lie along a line $\Psi=$ constant. Thus, as shown in figure 3, the interior of half the flow field must be mapped into an infinite strip in the $\mathrm{W}$-plane. Now, the $\mathrm{t}$-plane is chosen so that the boundaries of the flow field are as shown in figure 4; that is, the bounding surface of the arc is

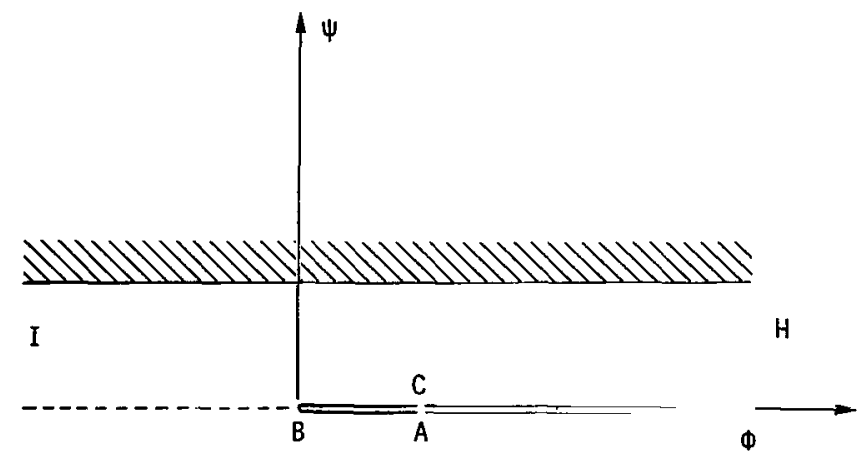

Figure 3. - W-plane.

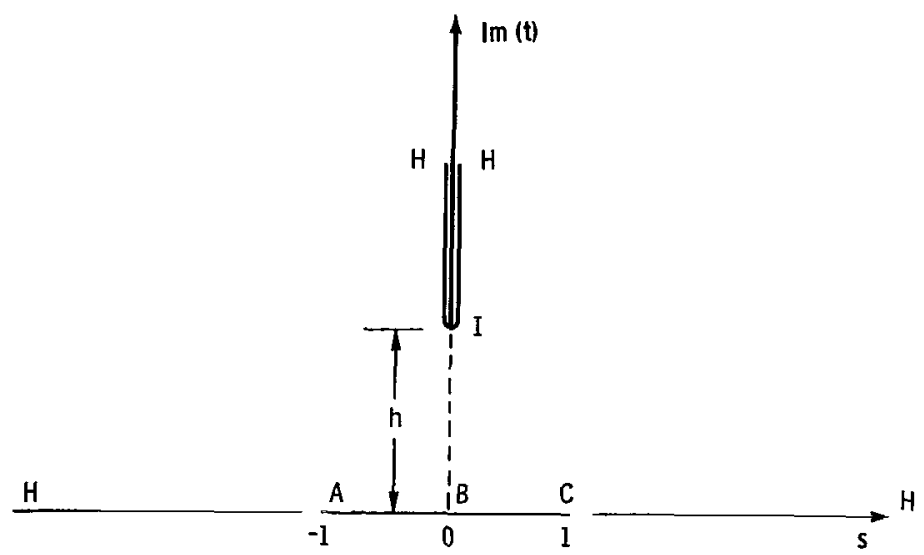

Figure 4. - t-Plane. 
mapped into the interval $(-1,1)$ on the real axis; the bounding streamlines of the wake are mapped into the rest of the real axis, the channel walls are mapped into the portion of the imaginary axis above the point I; and the stagnation streamline is mapped into the rest of the positive imaginary axis. The point at upstream infinity in the physical z-plane is mapped into the point $I$ on the imaginary axis, and the point far downstream in the physical plane is mapped into the point at infinity in the $t$-plane.

A mapping must be found of the interior of the infinite strip in the W-plane into the first quadrant of the $t$-plane. To accomplish this maping, note that the transformation

$$
T=t^{2}
$$

maps the first quadrant of the t-plane into the upper-half $T$-plane, as shown in figure 5, so that the positive imaginary axis in the t-plane is mapped into the negative real axis in the T-plane. Now, map the interior of the infinite strip in the W-plane into the upperhalf $T$-plane by the degenerate Schwarz-Christoffel transformation (ref. 3, p. 226)

$$
\frac{d W}{d T}=\frac{\Psi_{o}}{1-\left(\frac{T}{T_{I}}\right)}
$$

Eliminating $T$ between equations (10) and (11) and noting from figure 4 that, at the point $I$ in the $\mathrm{t}$-plane, $\mathrm{t} \equiv$ ih yield

$$
\frac{\mathrm{dW}}{\mathrm{dt}}=\frac{2 \Psi_{\mathrm{o}} \mathrm{t}}{1+\frac{\mathrm{t}^{2}}{\mathrm{~h}^{2}}}
$$

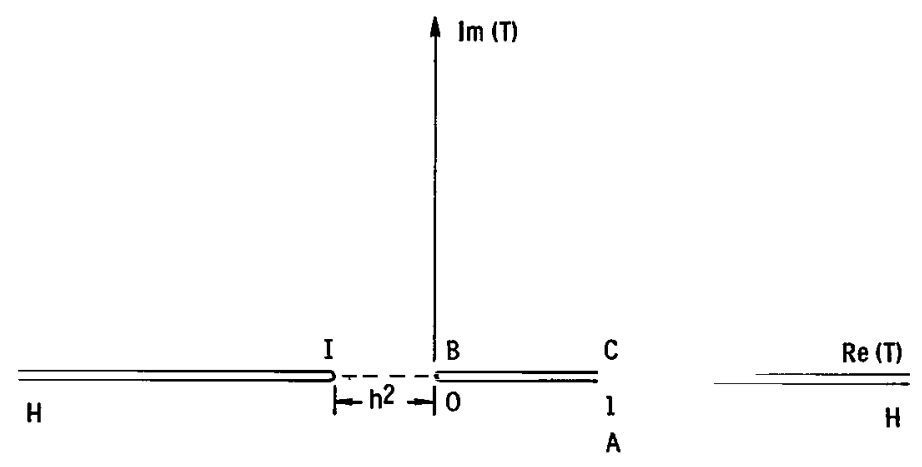

Figure 5. - T-plane. 
To establish the connection between $\Psi_{0}, h$, and the physical variables, integrate equation (12) to get

$$
W=h^{2} \Psi_{0} \ln \left(t^{2}+h^{2}\right)+\text { constant }
$$

Now, since $\mathrm{W}\left(-\mathrm{t}^{*}\right)=\mathrm{W}^{*}(\mathrm{t})$, it is clear from the symmetry of the problem that equation (13) gives the correct complex potential for the entire flow field when it is extended from the first quadrant of the $t$-plane to the upper-half $t$-plane. The imaginary part of $\mathrm{W}$ can be shown to change by $2 \pi \mathrm{h}^{2} \Psi_{\mathrm{O}}$ in passing from the negative to positive side of the imaginary axis with $\operatorname{Im}(t)>h$; that is, the change in $\Psi$ between the channel walls is $2 \pi \mathrm{h}^{2} \Psi_{\mathrm{o}}$. But this is the total flow through the channel and is ther efore equal to $\mathrm{V}_{\infty} \mathrm{D}$, where $V_{\infty}$ is the upstream velocity of the flow. Hence, it follows that

$$
V_{\infty} D=2 \pi h^{2} \Psi_{0}
$$

Equation (12) shows that, in the limit $\mathrm{h} \rightarrow \infty$,

$$
W=\Psi_{0} t^{2}
$$

which maps the entire $\mathrm{W}$-plane into the upper-half $\mathrm{t}$-plane. This expression is the proper transformation of the $\mathrm{W}$-plane into the $\mathrm{t}$-plane for an unbounded flow (channel walls infinitely far apart). Note that in this case the stagnation streamline maps into the entire positive imaginary axis. Hence, at least as far as $W$ is concerned, the limit $D \rightarrow \infty$ corresponds to the limit $h \rightarrow \infty$. The next section makes apparent the fact that $\zeta$ depends on $h$ only through $\mathrm{dW} / \mathrm{dt}$ and so the same conclusion follows for $\zeta$.

\section{Calculation of Velocity}

With $W$ specified as a function of the intermediate variable $t, \zeta$ is now found as a function of $t$. This is accomplished by studying the properties of the function $\eta$, defined by

$$
\eta(t)=\left(\frac{1+i \sqrt{t^{2}-1}}{t}\right)^{\beta} e^{i(1-\beta) \pi / 2} \quad 0<\beta \leq 1
$$


The branch of $\sqrt{t^{2}-1}$ is chosen so that the cut lies along the real axis between -1 and 1 (see ref. 4, p. 82). When $t$ approaches the real axis through values with positive imaginary part,

$$
\sqrt{t^{2}-1}=\left\{\begin{array}{cc}
i\left|1-t^{2}\right|^{1 / 2} & -1<t<1 \\
\left|t^{2}-1\right|^{1 / 2} & t>1 \\
-\left|t^{2}-1\right|^{1 / 2} & t<-1
\end{array}\right.
$$

and

$$
\lim _{\epsilon \rightarrow 0+} \eta(t+i \epsilon)= \begin{cases}\left(\frac{|t|}{1+\left|1-t^{2}\right|^{1 / 2}}\right)^{\beta} e^{i(1-\beta) \pi / 2} & 0<t<1 \\ \left(\frac{|t|}{1+\left|1-t^{2}\right|^{1 / 2}}\right)^{\beta} e^{i(1+\beta) \pi / 2} & -1<t<0\end{cases}
$$

It follows that

$$
\begin{aligned}
& \lim _{\epsilon \rightarrow 0} \arg \eta(t+i \epsilon)= \begin{cases}(1-\beta) \pi / 2 & 0<t<1 \\
(1+\beta) \pi / 2 & -1<t<0\end{cases} \\
& \eta(t)= \begin{cases}e^{i\left[(1-\beta) \pi / 2+\beta \tan ^{-1} \sqrt{t^{2}-1}\right]} & t>1 \\
i\left[(1-\beta) \pi / 2+\beta \tan ^{-1}\left(-\sqrt{t^{2}-1}\right)\right] & t<-1 \\
e & t\end{cases}
\end{aligned}
$$

where the arc tangent must lie in the first and second quadrants. Also 


$$
|\eta(t)|=1 \quad t<-1, t>1
$$

so that $\arg \eta(t)$ goes from $(1-\beta) \pi / 2$ to $\pi / 2$ as $t$ goes from 1 to $\infty$ along the real axis and from $(1+\beta) \pi / 2$ to $\pi / 2$ as $t$ goes from -1 to $-\infty$ along the real axis. Note, also, that when $t=i \lambda$ for $\lambda>0$,

$$
\eta(i \lambda)=i\left(\frac{\lambda}{\sqrt{\lambda^{2}+1}+1}\right)^{\beta} \quad \lambda>0
$$

Thus,

$$
\arg \eta(i \lambda)=\frac{\pi}{2} \quad \lambda>0
$$

In fact, $\lim _{t \rightarrow \infty} \arg \eta(t)=\pi / 2$ everywhere in the upper-half plane, or

$$
\lim _{t \rightarrow \infty} \eta(t)=e^{i \pi / 2}
$$

Now consider $\zeta$. It is not known yet whether the boundary of the arc has a finite curvature at the stagnation point $B$ or whether a sharp angle occurs at this point. There is no reason to exclude the latter case from consideration. Define

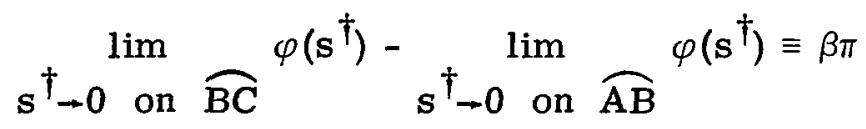

that is, $\beta \pi$ is the included angle between the two tangents to the arc surface at the stagnation point (see fig. 2).

A classical result (ref. 4, p. 132) in conformal mapping is that in the vicinity of $\mathrm{t}=0, \mathrm{dt} / \mathrm{dz} \sim \mathrm{t}^{\beta-1}$. Because

$$
\zeta=\frac{d W}{d z}=\frac{d w}{d t} \frac{d t}{d z}
$$

it follows, by using equation (12), that $\zeta \sim \mathrm{t}^{\beta}$ in the vicinity of $t=0$, which shows that, unless the arc has a cusp $(\beta=0)$ at the stagnation point, $\zeta$ vanishes there as $t^{\beta}$. It is also known from the nature of the problem that $\zeta \neq 0, \infty$ at any other point. Since the 
velocity in the channel must become uniform far downstream of the arc, it follows from figures 2 and 4 that, in the upper-half $t$-plane,

$$
\lim _{t \rightarrow \infty} \zeta(t)=v_{0} e^{i \pi / 2}
$$

Equation (15) shows that $\eta(t) \sim t^{\beta}$ in the vicinity of $t=0$ and $\eta(t) \neq 0$, $\infty$ at any other point in the upper-half $t$-plane. Also, equation (19) holds as $t-\infty$.

The interior of the first quadrant of the t-plane is denoted by $\Gamma$ (i. e., the first quadrant of the t-plane excluding the positive real and imaginary axes), and the closure of $\Gamma$ (that is, the first quadrant of the $t$-plane including the positive real and imaginary axes) is dented by $\bar{\Gamma}$. It is clear that half the flow (that is, the region in $\mathrm{IBC}$ HI of fig. 2) is mapped into $\overline{\boldsymbol{\Gamma}}$.

From the preceding discussion, the function $\zeta(t) / \mathrm{v}_{\mathrm{o}} \eta(\mathrm{t})$ for $t \in \bar{\Gamma}$ is bounded away from 0 and $\infty$ in $\bar{\Gamma}$, is analytic in $\Gamma$, and approaches unity as $t \rightarrow \infty$ for all $t \in \bar{\Gamma}$.

Introduce a new function of $\mathrm{t}, \Omega=\theta+\tau(\theta$ and $\tau$ real) defined by

$$
\zeta(\mathrm{t})=\mathrm{V}_{\mathrm{o}} \eta(\mathrm{t}) \mathrm{e}^{-\mathrm{i} \Omega(\mathrm{t})}
$$

Then, $\Omega$ is analytic in $\Gamma$ and bounded, and turns out to be continuous in $\bar{\Gamma}$ (ref. 5 , Theorem 14.19, p. 281 and Remarks $14.20 \mathrm{C}$, p. 282). Also, since

$$
\ln \frac{\zeta(t)}{v_{0} \eta(t)}=-i \Omega(t) \quad t \in \bar{\Gamma}
$$

it follows from equations (20) and (21), that

$$
\lim _{t \rightarrow \infty} \Omega(t)=0 \quad t \in \bar{\Gamma}
$$

Also, figures 2 and 4 clearly show that $\zeta$ must have the argument $\pi / 2$ on the positive imaginary axis of the $t$-plane. Since

$$
\arg \zeta=\arg \eta-\theta
$$

it is clear from equation (19), $\theta=0$ on the positive imaginary axis; that is, $\Omega$ is a pure imaginary quantity on the positive imaginary axis. Then, by Schwarz's Principle of Reflection (ref. 4, pp. 44 or ref. 5, Theorem 11.17, p. 230), $\Omega(t)$ can be extended ana- 
lytically to a function analytic in the entire upper-half plane (excluding the real axis) by

$$
\Omega\left(-t^{*}\right)=-\Omega *(t)
$$

Equations (15) and (24) show that $\zeta(t)$ given by equation (22) then has the proper symmetry in the upper-half $t$-plane to describe the flow depicted in figure 2. Inasmuch as the free streamlines bounding the wake are mapped into the portion of the real axis outside the interval $(-1,1)$ (fig. 4), it can be concluded that $|\zeta(t)|=V_{0}$ for $t<-1$ and $t>1$. Equation (22) shows that

$$
|\zeta(t)|=v_{0}|\eta(t)| e^{\tau(t)}
$$

thus, from equation (17a),

$$
\mathrm{e}^{\tau(\mathrm{t})}=1 \quad \mathrm{t}<-1, \mathrm{t}>1
$$

which shows that

$$
\tau(t)=0 \quad t \leq-1, t \geq 1
$$

The complex conjugate velocity $\zeta(t)$, given by equation (22) with the symmetry condition (eq. (24)) and the conditions of equations (23a) and (26), now satisfies all the specified velocity boundary conditions except those on the arc surface corresponding to the interval $(-1,1)$ in the $t$-plane. Equations $(23)$ and $(24)$ show that

$$
\lim _{t \rightarrow \infty} \Omega(t)=0
$$

along all paths in the upper-half plane.

Since $\Omega(t)$ is analytic in the upper-half plane, vanishes at infinity, and is bounded on the real axis, $\Omega(t)$ may be expressed everywhere in the upper-half plane in terms of its imaginary part on the real axis by using the Schwarz integral formula (ref. 3, p. 251), namely,

$$
\Omega(t)=\frac{1}{\pi} \int_{-\infty}^{\infty} \frac{\tau(s)}{s-t} d s
$$


where

$$
\mathrm{s} \equiv \operatorname{Re}(\mathrm{t})
$$

By use of equation (26), the Schwarz formula becomes

$$
\Omega(t)=\frac{1}{\pi} \int_{-1}^{1} \frac{\tau(s)}{s-t} d s
$$

It is easily seen that the function $\Omega$, given by equation (28) satisfies equations (23b) and (26) and that the condition

$$
\tau(-\mathrm{s})=\tau(\mathrm{s})
$$

is necessary and sufficient for equation (24) to hold (which also guarantees that $\Omega$ be a pure imaginary quantity on the positive imaginary axis).

Observe that $\zeta(t)$, given by equations (22) and (28) with the symmetry condition (eq. (29)), satisfies all the boundary conditions on the velocity except the one on the boundary of the $\operatorname{arc}(-1,1)$ in the t-plane.

In order to find $\theta(\mathrm{s})$ for $-1 \leq \mathrm{s} \leq 1$ from equation (28), the Plemelj formulas (also called Sohkosti formulas) for the limiting value of an analytic function (ref. 6, p. 25) are used. Corresponding to equation (28), the Plemelj formula appropriate to the upper-half $\mathrm{t}$-plane is

$$
\begin{aligned}
\lim _{\mathrm{t} \rightarrow \mathrm{s}+} \Omega(\mathrm{t}) & =\theta(\mathrm{s})+\mathrm{i} \tau(\mathrm{s}) \\
& =\mathrm{i} \tau(\mathrm{s})+\text { P. v. } \frac{1}{\pi} \int_{-1}^{1} \frac{\tau\left(\mathrm{s}^{\prime}\right)}{\mathrm{s}^{\prime}-\mathrm{s}} \mathrm{ds}^{\prime} \quad-1<\mathrm{s}<1
\end{aligned}
$$

where $t \rightarrow s+$ indicates that $t$ approaches the real axis through values with positive imaginary parts, P. V. shows that the singular integral to the right is to be evaluated in the sense of a Cauchy Principal Value, and $s^{\prime}$ is a dummy variable of integration. Thus, $\theta(s)$ is given on the interval $(-1,1)$ in terms of the values of $\tau$ on this interval by

$$
\theta(\mathrm{s})=\text { P. V. } \frac{1}{\pi} \int_{-1}^{1} \frac{\tau\left(\mathrm{s}^{\prime}\right)}{\mathrm{s}^{\prime}-\mathrm{s}} \mathrm{d \textrm {s } ^ { \prime }} \quad-1<\mathrm{s}<1
$$


Now, to complete the solution of the problem requires only the determination of $\tau$ on the real interval $(-1,1)$, for which equation (6) is used. First, note that the element of length $\mathrm{ds}^{\dagger}$ is given by

$$
\mathrm{ds}^{\dagger}=|\mathrm{dz}|
$$

where $d z$ is to be taken along the real interval $-1 \leq t \leq 1$ in the $t$-plane, since $s^{\dagger}$ maps into this interval. Now, because

$$
d z=\frac{d z}{d W} \frac{d W}{d t} d t=\zeta^{-1}\left(\frac{d W}{d t}\right) d t
$$

it follows that

$$
d s^{\dagger}=\left|\zeta^{-1}(s)\right|\left|\frac{d W}{d t}\right|_{t=s}|d s| \quad-1<s<1
$$

In general,

$$
\left|\zeta^{-1}(\mathrm{~s})\right|=|\zeta(\mathrm{s})|^{-1}
$$

and, by definition,

$$
\mathrm{v}_{\mathrm{s}}=|\zeta(\mathrm{s})|
$$

Using these results in equation (6) gives

$$
\left.\begin{array}{c}
\rho \mathrm{v}_{\mathrm{s}}^{2} \mathrm{dV}_{\mathrm{s}}=\mathrm{jB} \cos \varphi\left|\frac{\mathrm{dW}}{\mathrm{dt}}\right|_{\mathrm{t}=\mathrm{s}}|\mathrm{ds}| \\
\mathrm{V}_{\mathrm{S}}=|\zeta(\mathrm{s})|
\end{array}\right\}-1 \leq \mathrm{s} \leq 1
$$

The surface of the arc is a streamline (see fig. 2); hence, 


$$
\arg \zeta^{-1}(s)=\left\{\begin{array}{cc}
\varphi-\frac{\pi}{2} & 0<s \leq 1 \\
-\varphi-\frac{\pi}{2} & -1 \leq s<0
\end{array}\right.
$$

but from equation (22),

$$
\arg \zeta^{-1}(s)=\theta-\arg \eta(s) \quad-1 \leq s \leq 1
$$

Using equation (16a) yields

$$
\arg \zeta^{-1}(s)= \begin{cases}\theta-(1-\beta) \pi / 2 & 0<s \leq 1 \\ \theta-(1+\beta) \pi / 2 & -1 \leq s<0\end{cases}
$$

so that

$$
\varphi=\left\{\begin{array}{cc}
\theta+\frac{\beta \pi}{2} & 0<\mathrm{s} \leq 1 \\
-\theta+\frac{\beta \pi}{2} & -1 \leq \mathrm{s}<0
\end{array}\right.
$$

Integrating equation (31) from 0 to $s(-1<s<1)$ and noting that $V_{s}$ is zero at $s=0$ (neglecting here the case of a cusp $(\beta=0)$ at the stagnation point) yield

$$
\begin{aligned}
|\zeta(s)|^{3} & \equiv v_{s}^{3} \\
& =\frac{3 j B}{\rho} \int_{0}^{s} \cos \varphi\left(s^{\prime}\right)\left|\frac{d w}{d t}\right|_{t=s^{\prime}}\left|d^{\prime}\right| \quad-1 \leq s \leq 1
\end{aligned}
$$

From equations (16) and (25), 


$$
\begin{aligned}
|\zeta(\mathrm{s})| & =\mathrm{v}_{\mathrm{o}}|\eta(\mathrm{s})| \mathrm{e}^{\tau(\mathrm{s})} \\
& =\mathrm{v}_{0}\left(\frac{|\mathrm{s}|}{1+\sqrt{1-\mathrm{s}^{2}}}\right)^{\beta} \mathrm{e}^{\tau(\mathrm{s})} \quad-1 \leq \mathrm{s} \leq 1
\end{aligned}
$$

Substituting equations (12), (32), and (34) into equation (33) gives

$$
\left(\frac{s}{1+\sqrt{1-s^{2}}}\right)^{3 \beta} e^{3 \tau(s)}=\frac{6 j B \Psi_{o}}{\rho V_{o}^{3}} \int_{0}^{s} \cos \left[\theta\left(s^{\prime}\right)+\frac{\beta \pi}{2}\right] \frac{s^{\prime}}{1+\left(s^{\prime}\right)^{2} / h^{2}} d s^{\prime} \quad 0 \leq s \leq 1
$$

Setting $s=1$ in equation (35) and using equation (26) show that

$$
\frac{\rho \mathrm{V}_{0}^{3}}{6 j \mathrm{~B} \Psi_{0}}=\int_{0}^{1} \cos \left(\theta+\frac{\beta \pi}{2}\right) \frac{\mathrm{s}}{1+\mathrm{s}^{2} / \mathrm{h}^{2}} \mathrm{ds}
$$

Eliminating $6 \mathrm{jB} \Psi_{\mathrm{o}} / \rho \mathrm{V}_{\mathrm{o}}^{3}$ between equations (35) and (36) leads to

$$
\left(\frac{\mathrm{s}}{1+\sqrt{1-\mathrm{s}^{2}}}\right)^{3 \beta} \mathrm{e}^{3 \tau(\mathrm{s})}=\frac{\int_{0}^{\mathrm{s}} \cos \left[\theta\left(\mathrm{s}^{\prime}\right)+\frac{\beta \pi}{2}\right] \frac{\mathrm{s}^{\prime}}{1+\left(\mathrm{s}^{\prime}\right)^{2} / \mathrm{h}^{2}} \mathrm{ds^{ \prime }}}{\int_{0}^{1} \cos \left(\theta+\frac{\beta \pi}{2}\right) \frac{\mathrm{s}}{1+\mathrm{s}^{2} / \mathrm{h}^{2}} \mathrm{ds}} \quad 0 \leq \mathrm{s} \leq 1
$$

From equations (29) and (30) or equivalently from equation (24),

$$
\theta(-s)=-\theta(s) \quad 0 \leq s \leq 1
$$

so that $\theta$ is an odd function of $s$ on the interval $(-1,1)$. In particular,

$$
\theta(0)=0
$$


Equation (39) shows that in the neighborhood of $s=0$

$$
\cos \left(\theta+\frac{\beta \pi}{2}\right) \sim \cos \frac{\beta \pi}{2} \quad \beta \neq 1
$$

and

$$
\cos \left(\theta+\frac{\beta \pi}{2}\right)=-\sin \theta \sim \theta \sim s \quad \beta=1
$$

so that, in the neighborhood of $s=0$, the right side of equation (37) behaves like $s^{2}$ for $\beta \neq 1$, whereas the left side behaves like $s^{3 \beta}$. It can be concluded from this that solutions to equation (37), which are continuous in the neighborhood of $s=0$, exist only if $\beta=1$ or $\beta=2 / 3$. Thus, the arc surface is either a smooth curve $(\beta=1)$ in the neighborhood of the stagnation point, or else it is pointed with an included angle of $120^{\circ}$ (compare with $120^{\circ}$ wedge solution of ref. 2). Solving equation (37) for $\tau$ yields

$$
\begin{aligned}
& \tau(\mathrm{s})=\beta \ln \left(1+\sqrt{1-\mathrm{s}^{2}}\right)+\frac{1}{3} \ln \left[\frac{1}{\mathrm{~s}^{3 \beta}} \frac{\int^{\mathrm{s}} \cos \left(\theta+\frac{\beta \pi}{2}\right) \frac{\mathrm{s}}{1+\mathrm{s}^{2} / \mathrm{h}^{2}} \mathrm{ds}}{\int_{0}^{1} \cos \left(\theta+\frac{\beta \pi}{2}\right) \frac{\mathrm{s}}{1+\mathrm{s}^{2} / \mathrm{h}^{2}} \mathrm{ds}}\right] \\
& 0 \leq \mathrm{s} \leq 1 ; \beta=1,2 / 3
\end{aligned}
$$

The problem is now essentially solved since the $\tau$ given by equation (40) for positive $\mathrm{S}$ can be extended to negative values of $s$ by the symmetry condition (eq. (29)). The result can be substituted in equation (30). In this manner, a nonlinear integral equation for $\theta$ is obtained, and the solution to this equation can be obtained numerically. Equations (40) and (29) can be used to calculate $\tau(\mathrm{s})$ for $-1 \leq \mathrm{s} \leq-1$. These values of $\tau$ can then be substituted into equation (28) to determine $\Omega$ in the upper-half t-plane and, therefore, by equation (22) to determine the complex conjugate velocity $\zeta(t)$. Thus, knowing $\mathrm{W}$ from equation (13) and $\zeta$ allows the calculation of all physical quantities, as explained in the discussion of equation (9) (see the Calculation of Stream Function section). The substitutions just described do not have to be carried out, since the numerical solutions to equations (29), (30), and (40) were obtained with the equations in the form in which they are now written. The solutions for an arc in an unbounded free stream can 
be obtained by letting $h \rightarrow \infty$ in equation (40).

Before the results are collected and summarized, the expressions for the most important physical variables must be developed (the arc shape and the shape of the free streamlines), and the relations between the parameter $h$ and the physical quantities of interest in the problem must be found.

\section{Boundaries of Arc and Wake}

Expressions for the shape of the bounding streamlines are obtained first. Notice that the surface of the arc $\overparen{\mathrm{BC}}$ in figure 2 is mapped into the interval $0 \leq \mathrm{s} \leq 1$ in the $t$-plane. Hence, the position of the arc surface, denoted by $z_{s}=x_{s}+i y_{s}$, is given by equation (9) as

$$
z_{s}=\int_{0}^{s} \zeta^{-1}(s)\left(\frac{d w}{d t}\right)_{t=s} \text { ds } \quad 0 \leq s \leq 1
$$

where the origin of the coordinate system lies at the stagnation point. Substituting equations (12), (16), and (22) into equation (41) yields

$$
z_{s}=\frac{2 \Psi_{0}}{v_{0}} \int_{0}^{s} e^{i[\theta-(1-\beta) \pi / \beta]} e^{-\tau}\left(\frac{s}{1+\sqrt{1-s^{2}}}\right)^{-\beta} \frac{s}{1+s^{2} / h^{2}} d s
$$

The real and imaginary parts of $z_{s}$ are

$$
\mathrm{x}_{\mathrm{s}}=\frac{2 \Psi_{0}}{\mathrm{v}_{\mathrm{o}}} \int_{0}^{\mathrm{s}} \cos [\theta-(1-\beta) \pi / 2] \mathrm{e}^{-\tau}\left(\frac{\mathrm{s}}{1+\sqrt{1-\mathrm{s}^{2}}}\right)^{-\beta} \frac{\mathrm{s}}{1+\mathrm{s}^{2} / \mathrm{h}^{2}} \mathrm{ds}
$$

and 


$$
\begin{aligned}
\mathrm{y}_{\mathrm{s}} & =\frac{2 \Psi_{0}}{\mathrm{v}_{\mathrm{o}}} \int_{0}^{\mathrm{s}} \sin [\theta-(1-\beta) \pi / 2] \mathrm{e}^{-\tau}\left(\frac{\mathrm{s}}{1+\sqrt{1-\mathrm{s}^{2}}}\right)^{-\beta} \frac{\mathrm{s}}{1+\mathrm{s}^{2} / \mathrm{h}^{2}} \mathrm{ds} \\
& =-\frac{2 \Psi_{0}}{\mathrm{v}_{\mathrm{o}}} \int_{0}^{\mathrm{s}} \cos \left(\theta+\frac{\beta \pi}{2}\right) \mathrm{e}^{-\tau}\left(\frac{\mathrm{s}}{1+\sqrt{1-\mathrm{s}^{2}}}\right)^{-\beta} \frac{\mathrm{s}}{1+\mathrm{s}^{2} / \mathrm{h}^{2}} \mathrm{ds}
\end{aligned}
$$

This last expression can be simplified further. First, differentiate both sides of equation (35) with respect to $s$ to get

$$
\left(\frac{\mathrm{s}}{1+\sqrt{1-\mathrm{s}^{2}}}\right)^{2 \beta} \mathrm{e}^{2 \tau} \frac{\mathrm{d}}{\mathrm{ds}}\left(\frac{\mathrm{s}}{1+\sqrt{1-\mathrm{s}^{2}}}\right)^{\beta} \mathrm{e}^{\tau}=\frac{2 \mathrm{jB \Psi} \mathrm{o}}{\rho \mathrm{V}_{\mathrm{o}}^{3}} \cos \left(\theta+\frac{\beta \pi}{2}\right) \frac{\mathrm{s}}{1+\mathrm{s}^{2} / \mathrm{h}^{2}}
$$

Using this expression to eliminate $\cos (\theta+\beta \pi / 2)$ in equation (43) gives

$$
\begin{aligned}
\mathrm{y}_{\mathrm{s}} & =-\frac{\rho \mathrm{v}_{\mathrm{o}}^{2}}{2 \mathrm{jB}} \int_{0}^{\mathrm{s}} \frac{\mathrm{d}}{\mathrm{ds}}\left(\frac{\mathrm{s}}{1+\sqrt{1-\mathrm{s}^{2}}}\right)^{2 \beta} \mathrm{e}^{2 \tau} \mathrm{ds} \\
& =-\frac{\rho \mathrm{v}_{\mathrm{o}}^{2}}{2 \mathrm{jB}}\left(\frac{\mathrm{s}}{1+\sqrt{1-\mathrm{s}^{2}}}\right)^{2 \beta} \mathrm{e}^{2 \tau}
\end{aligned}
$$

Denote the longest dimension of the arc in the direction parallel to the flow by $d$; thus, $\mathrm{y}_{\mathrm{s}}=-\mathrm{d}$ at $\mathrm{s}=1$. From equation (26), $\tau(1)=0$, so that equation (44) shows

$$
\mathrm{d}=\frac{\rho \mathrm{V}_{\mathrm{o}}^{2}}{2 \mathrm{jB}}
$$

or 


$$
\frac{\mathrm{jBd}}{\frac{1}{2} \rho \mathrm{V}_{\mathrm{o}}^{2}}=1
$$

which is the equilibrium condition satisfied by the parameters of the magnetically balanced arc. Using equations (45) and (36) in equation (42) finally leads to

$$
\frac{\mathrm{x}_{\mathrm{s}}}{\mathrm{d}}=\frac{2}{3} \frac{\cos [\theta-(1-\beta) \pi / 2] \mathrm{e}^{-\tau} \frac{\left(1+\sqrt{1-\mathrm{s}^{2}}\right)^{\beta}}{1+\mathrm{s}^{2} / \mathrm{h}^{2}} \mathrm{~s}^{1-\beta} \mathrm{ds}}{\int_{0}^{1} \cos \left(\theta+\frac{\beta \pi}{2}\right) \frac{\mathrm{s}}{1+\mathrm{s}^{2} / \mathrm{h}^{2}} \mathrm{ds}} \quad 0 \leq \mathrm{s} \leq 1
$$

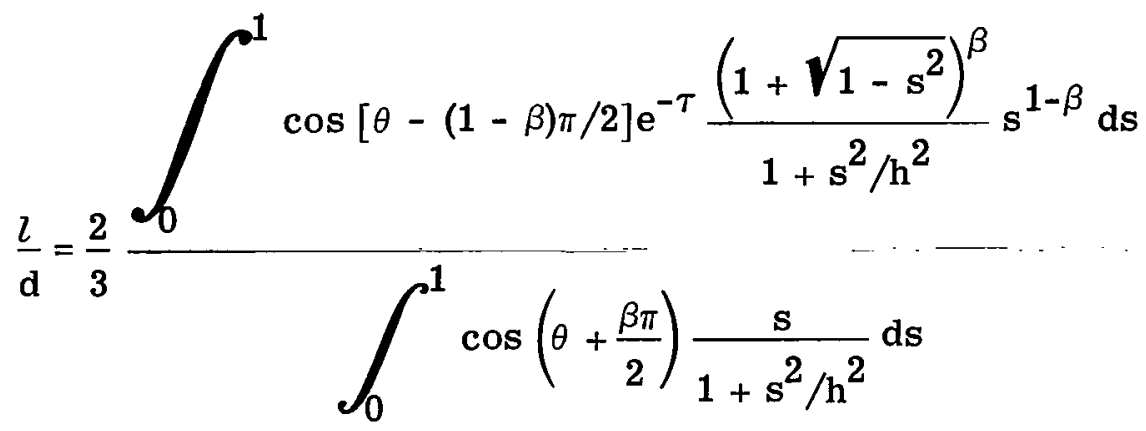

and rewriting equation (44) in terms of $d$ yields

$$
\frac{y_{s}}{d}=-\left(\frac{s}{1+\sqrt{1-s^{2}}}\right)^{2 \beta} e^{2 \tau(s)} \quad 0 \leq s \leq 1
$$

Having obtained a set of parametric equations for the bounding surface of the arc, the next step is to obtain an expression for the boundary of the wake. Notice that the bounding streamline of the wake $\overparen{C H}$ in figure 2 is mapped into the interval $s \geq 1$ in the $t$-plane. Hence, the position of the wake surface defined as $z_{f}=x_{f}+i y_{f}$ is given by 


$$
z_{f}=\left(z_{s}\right)_{s=1}+\int_{1}^{s} \zeta^{-1}(s)\left(\frac{d W}{d t}\right)_{t=s} d s \quad s \geq 1
$$

Substituting equations (12), (17), (22), and (26) into equation (48) leads to

$$
z_{f}=\left(z_{s}\right)_{s=1}+\frac{2 \Psi_{0}}{v_{0}} \int_{1}^{s} e^{i\left[\theta-(1-\beta) \pi / 2-\beta \tan ^{-1} \sqrt{s^{2}-1}\right]} \frac{s}{1+s^{2} / h^{2}} d s \quad s \geq 1
$$

The real and imaginary parts of $z_{f}$ are

$$
\mathrm{x}_{\mathrm{f}}=l+\frac{2 \Psi_{\mathrm{o}}}{\mathrm{v}_{\mathrm{o}}} \int_{1}^{\mathrm{s}} \cos \left[\theta-(1-\beta) \pi / 2-\beta \tan ^{-1} \sqrt{\mathrm{s}^{2}-1}\right] \frac{\mathrm{s}}{1+\mathrm{s}^{2} / \mathrm{h}^{2}}
$$

and

$$
\left.\mathrm{y}_{\mathrm{f}}=-\mathrm{d}+\frac{2 \Psi_{\mathrm{o}}}{\mathrm{v}_{\mathrm{o}}} \int_{1}^{\mathrm{s}} \sin \left[\theta-(1-\beta) \pi / 2-\beta \tan ^{-1} \sqrt{\mathrm{s}^{2}-1}\right] \frac{\mathrm{s}}{1+\mathrm{s}^{2} / \mathrm{h}^{2}} \mathrm{ds}\right]
$$

where $l$ is given by equation (46b). Using equations (45) and (36) in equation (49) gives

$$
\frac{\mathrm{x}_{\mathrm{f}}}{\mathrm{d}}=\frac{l}{\mathrm{~d}}+\frac{2}{3} \frac{\int_{1}^{\mathrm{s}} \cos \left[\theta-(1-\beta) \pi / 2-2 \tan ^{-1} \sqrt{\mathrm{s}^{2}-1}\right] \frac{\mathrm{s}}{1+\mathrm{s}^{2} / \mathrm{h}^{2}} \mathrm{ds}}{\int_{0}^{1} \cos \left(\theta+\frac{\beta \pi}{2}\right) \frac{\mathrm{s}}{1+\mathrm{s}^{2} / \mathrm{h}^{2}} \mathrm{ds}} \mathrm{s} \geq 1
$$




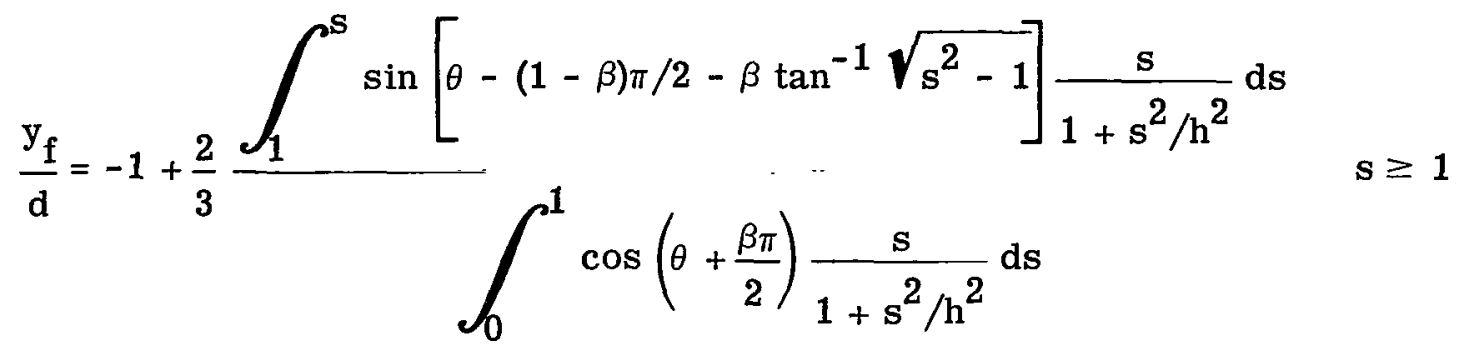

Equations (46a) and (47) and equations (50) and (51) give the shape of the arc and wake, respectively. Taking the limit $h-\infty$ in these equations gives the results for an arc in an unbounded free stream.

\section{Physical Parameters}

To obtain an expression for $\mathrm{V}_{\infty} / \mathrm{V}_{\mathrm{o}}$ in terms of $\mathrm{h}$, note that far upstream of the arc $\zeta=\mathrm{iV}_{\infty}$. In the $t$-plane, this point corresponds to $t=i$, and so equation (22) shows that

$$
i V_{\infty}=V_{o} \eta(i h) \mathrm{e}^{i \Omega(i h)}
$$

but from equation (28),

$$
\begin{aligned}
\Omega(\mathrm{ih}) & =\frac{1}{\pi} \int_{-1}^{1} \frac{\tau(\mathrm{s})}{\mathrm{s}-\mathrm{ih}} \mathrm{ds} \\
& =\frac{1}{\pi} \int_{-1}^{1} \frac{\mathrm{s} \tau(\mathrm{s})}{\mathrm{s}^{2}+\mathrm{h}^{2}} \mathrm{ds}+\frac{\mathrm{ih}}{\pi} \int_{-1}^{1} \frac{\tau(\mathrm{s})}{\mathrm{s}^{2}+\mathrm{h}^{2}} \mathrm{ds}
\end{aligned}
$$

Now equation (29) shows that $\tau(\mathrm{s}) /\left(\mathrm{s}^{2}+\mathrm{h}^{2}\right)$ is an even function of $\mathrm{s}$, and thus $s \tau(s) /\left(s^{2}+h^{2}\right)$ is an odd function of $s$. The first integral on the right of equation (53) is, therefore, zero. Introducing equations (18) and (53) into equation (52) yields

$$
\frac{v_{\infty}}{v_{o}}=\left(\frac{h}{\sqrt{h^{2}+1}+1}\right)^{\beta} e^{\frac{1}{\pi h}} \int_{-1}^{1} \frac{\tau(s)}{1+s^{2} / h^{2}} d s
$$


Equation (54) shows that in the limit $h-\infty, V_{0}=V_{\infty}$. Thus, in the case of an unbounded arc, the velocity far upstream must be equal to the velocity on the boundary streamline of the wake (or the velocity far downstream), which could have been anticipated beforehand from known results about unbounded flows. The characteristic dimension of the arc d can be related to the channel width $D$ by first eliminating $\Psi_{0}$ between equations (14) and (36) to yield

$$
\frac{\rho \mathrm{V}_{\mathrm{o}}^{3} \pi \mathrm{h}^{2}}{3 \mathrm{jB} \mathrm{V}_{\infty} \mathrm{D}}=\int_{0}^{1} \cos \left(\theta+\frac{\beta \pi}{2}\right) \frac{\mathrm{s}}{1+\mathrm{s}^{2} / \mathrm{h}^{2}} \mathrm{ds}
$$

Using equation (45) gives

$$
\frac{\mathrm{d}}{\mathrm{D}} \frac{\mathrm{v}_{\mathrm{o}}}{\mathrm{V}_{\infty}}=\frac{3}{2 \pi \mathrm{h}^{2}} \int_{0}^{1} \cos \left(\theta+\frac{\beta \pi}{2}\right) \frac{\mathrm{s}}{1+\mathrm{s}^{2} / \mathrm{h}^{2}} \mathrm{ds}
$$

or, with $V_{\infty} / V_{0}$ eliminated by equation (54),

$$
\frac{\mathrm{d}}{\mathrm{D}}=\frac{3}{2 \pi \mathrm{h}^{2}}\left(\frac{\mathrm{h}}{\sqrt{\mathrm{h}^{2}+1}+1}\right)^{\beta} \mathrm{e}^{\frac{1}{\pi \mathrm{h}}} \int_{-1}^{1} \frac{\tau(\mathrm{s})}{1+\mathrm{s}^{2} / \mathrm{h}^{2}} \mathrm{ds} \int_{0}^{1} \cos \left(\theta+\frac{\beta \pi}{2}\right) \frac{\mathrm{s}}{1+\mathrm{s}^{2} / \mathrm{h}^{2}} \mathrm{ds}
$$

which again shows that $D \rightarrow \infty$ when $\mathrm{h} \rightarrow \infty$.

The cross-sectional area $\mathrm{A}$ of the arc is given by

$$
\begin{aligned}
A & =-2 \int_{0}^{l} y_{s} d x_{s}=-2 d^{2} \int_{0}^{l / d}\left(\frac{y_{s}}{d}\right) d\left(\frac{x_{s}}{d}\right) \\
& =-2 d^{2} \int_{0}^{1}\left(\frac{y_{s}}{d}\right) \frac{d}{d s}\left(\frac{x_{s}}{d}\right) d s
\end{aligned}
$$

Substituting equations (46a) and (47) into the expression for $\mathrm{A}$ results in 


$$
A=\frac{4}{3} \mathrm{~d}^{2} \frac{\int_{0}^{1} \cos [\theta-(1-\beta) \pi / 2] \mathrm{e}^{\tau}\left(1-\sqrt{1-\mathrm{s}^{2}}\right)^{\beta} \frac{\mathrm{s}^{1-\beta}}{1+\mathrm{s}^{2} / \mathrm{h}^{2}} \mathrm{ds}}{\int_{0}^{1} \cos \left(\theta+\frac{\beta \pi}{2}\right) \frac{\mathrm{s}}{1+\mathrm{s}^{2} / \mathrm{h}^{2}} \mathrm{ds}}
$$

and equation $(46 \mathrm{~b})$ is used to get

$$
\frac{\mathrm{A}}{2 l \mathrm{~d}}=\frac{\int_{0}^{1} \cos [\theta-(1-\beta) \pi / 2] \mathrm{e}^{\tau}\left(1-\sqrt{1-\mathrm{s}^{2}}\right)^{\beta} \frac{\mathrm{s}^{1-\beta}}{1+\mathrm{s}^{2} / \mathrm{h}^{2}} \mathrm{ds}}{\int_{0}^{1} \cos [\theta-(1-\beta) \pi / 2] \mathrm{e}^{-\tau}\left(1+\sqrt{1-\mathrm{s}^{2}}\right)^{\beta} \frac{\mathrm{s}^{1-\beta}}{1+\mathrm{s}^{2} / \mathrm{h}^{2}} \mathrm{ds}}
$$

Combining equation (56) with equations (45) and (54) yields the drag coefficient

$$
\begin{aligned}
\frac{g \mathrm{~B}}{\left(\frac{1}{2} \rho \mathrm{v}_{\infty}^{2}\right)(2 \imath)}=\left(\frac{\sqrt{\mathrm{h}^{2}+1}+1}{\mathrm{~h}}\right)^{2 \beta} \mathrm{e}^{-\frac{2}{\pi \mathrm{h}}} \int_{-1}^{1} \frac{\tau(\mathrm{s})}{1+\mathrm{s}^{2} / \mathrm{h}^{2}} \mathrm{ds} \\
\qquad \int_{0}^{1} \cos [\theta-(1-\beta) \pi / 2] \mathrm{e}^{\tau}\left(1-\sqrt{1-\mathrm{s}^{2}}\right)^{\beta} \frac{\mathrm{s}^{1-\beta}}{1+\mathrm{s}^{2} / \mathrm{h}^{2}} \mathrm{ds} \\
\int_{0}^{1} \cos [\theta-(1-\beta) \pi / 2] \mathrm{e}^{-\tau}\left(1+\sqrt{1-\mathrm{s}^{2}}\right)^{\beta} \frac{\mathrm{s}^{1-\beta}}{1+\mathrm{s}^{2} / \mathrm{h}^{2}} \mathrm{ds}
\end{aligned}
$$

where $f=\mathrm{jA}$ is the total current in the arc.

\section{Summary of Calculated Quantities}

For convenience, the results are now collected. If $\beta=1$, the boundary of the arc is 
smooth at the stagnation point; if $\beta=2 / 3$, the boundary of the arc is pointed with $120^{\circ}$ included angle between the sides of the arc at the stagnation point. The parameter $\beta$ cannot have any other values. The following is a summary of the final results:

$$
\begin{gathered}
\tau(\mathrm{s})=\beta \ln \left(1+\sqrt{1-\mathrm{s}^{2}}\right)+\frac{1}{3} \ln \left[\frac{1}{\mathrm{~s}^{3 \beta}} \frac{\int_{0}^{\mathrm{s}} \cos \left(\theta+\frac{\beta \pi}{2}\right) \frac{\mathrm{s}}{1+\mathrm{s}^{2} / \mathrm{h}^{2}} \mathrm{ds}}{\int_{0}^{1} \cos \left(\theta+\frac{\beta \pi}{2}\right) \frac{\mathrm{s}}{1+\mathrm{s}^{2} / \mathrm{h}^{2}} \mathrm{ds}}\right] \\
0 \leq \mathrm{s} \leq 1 ; \beta=1,2 / 3 \\
\theta(\mathrm{s})=\text { P. V. } \frac{1}{\pi} \int_{-1}^{\frac{\tau\left(\mathrm{s}^{\prime}\right)}{\mathrm{s}^{\prime}-\mathrm{s}} \mathrm{d} \mathrm{s}^{\prime}} \quad-1<\mathrm{s}<1
\end{gathered}
$$

Equations (45) and (54) yield

$$
\begin{gathered}
\frac{j B d}{\frac{1}{2} \rho v_{\infty}^{2}}=\left(\frac{\sqrt{h^{2}+1}+1}{h}\right)^{2 \beta} e^{-\frac{2}{\pi h}} \int_{-1}^{1} \frac{\tau(s)}{1+s^{2} / h^{2}} d s \\
\frac{d}{D}=\frac{3}{2 \pi h^{2}}\left(\frac{h}{\sqrt{h^{2}+1}+1}\right)^{\beta} e^{\frac{1}{\pi h}} \int_{-1}^{\frac{\tau(s)}{1+s^{2} / h^{2}} d s} \int_{0}^{1} \cos \left(\theta+\frac{\beta \pi}{2}\right) \frac{s}{1+s^{2} / h^{2}} d s
\end{gathered}
$$




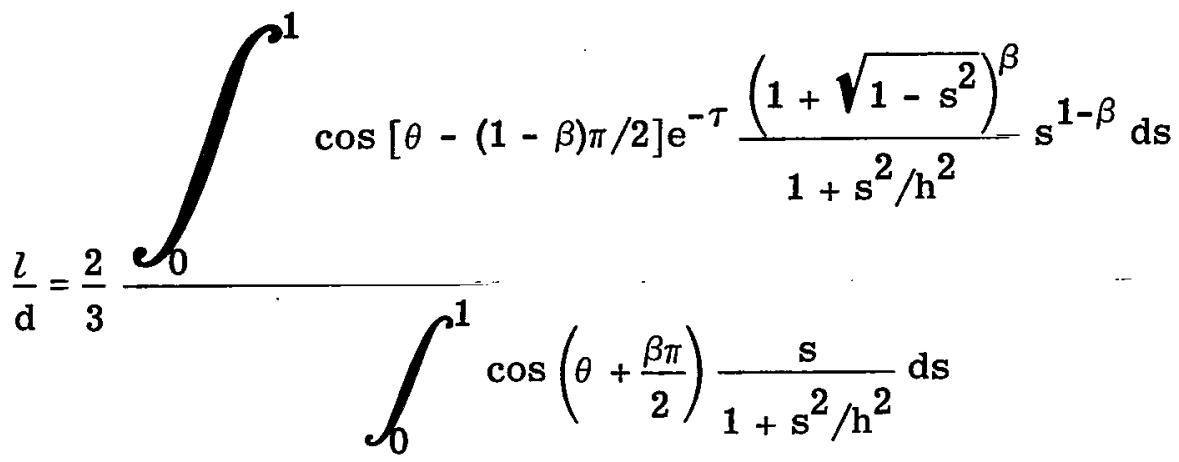

Taking the real part of equation (28) results in

$$
\theta(s)=\frac{1}{\pi} \int_{-1}^{1} \frac{\tau\left(s^{\prime}\right)}{s^{\prime}-s} d s^{\prime} \quad s>1, s<-1
$$

$$
\int_{\mathrm{d}}^{\mathrm{s}}=\frac{2}{3} \frac{\mathrm{cos}[\theta-(1-\beta) \pi / 2] \mathrm{e}^{-\tau} \frac{\left(1+\sqrt{1-\mathrm{s}^{2}}\right)^{\beta}}{1+\mathrm{s}^{2} / \mathrm{h}^{2}} \mathrm{~s}^{1-\beta} \mathrm{ds}}{\int_{0}^{1} \cos \left(\theta+\frac{\beta \pi}{2}\right) \frac{\mathrm{s}}{1+\mathrm{s}^{2} / \mathrm{h}^{2}} \mathrm{ds}} \quad 0 \leq \mathrm{s} \leq 1
$$

$$
\frac{y_{s}}{d}=-\left(\frac{s}{1+\sqrt{1-s^{2}}}\right)^{2 \beta} e^{2 \tau(s)} \quad 0 \leq s \leq 1
$$

$$
\frac{\mathrm{x}_{\mathrm{f}}}{\mathrm{d}}=\frac{l}{\mathrm{~d}}+\frac{2}{3} \frac{\int_{1}^{\mathrm{s}} \cos \left[\theta-(1-\beta) \pi / 2-\beta \tan ^{-1} \sqrt{\mathrm{s}^{2}-1}\right] \frac{\mathrm{s}}{1+\mathrm{s}^{2} / \mathrm{h}^{2}} \mathrm{ds}}{\int_{0}^{1} \cos \left(\theta+\frac{\beta \pi}{2}\right) \frac{\mathrm{s}}{1+\mathrm{s}^{2} / \mathrm{h}^{2}} \mathrm{ds}}
$$




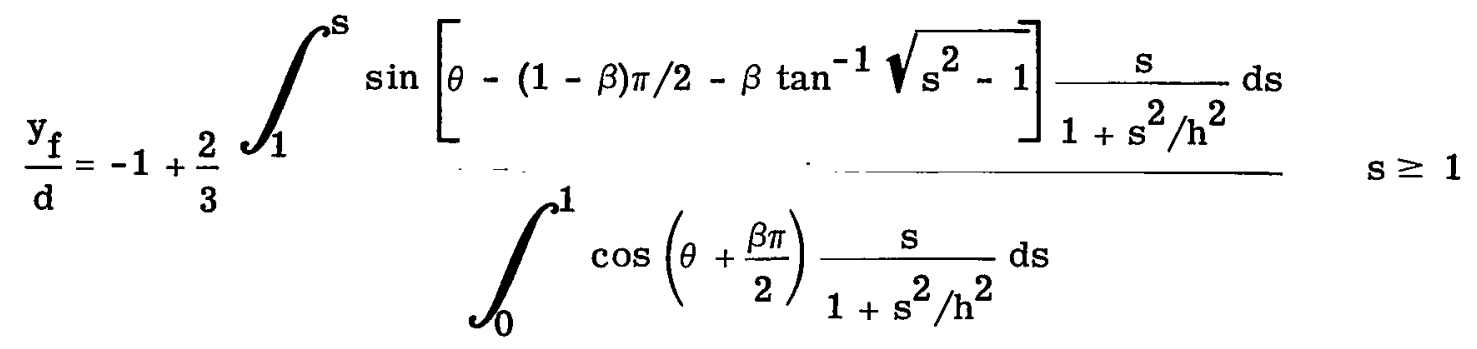

Finally, the drag coefficient $\mathscr{B} /\left(6 V_{\infty}^{2} / 2\right)(2 l)$ is

$$
\begin{aligned}
& \frac{s \mathrm{~B}}{\left(\frac{1}{2} \rho v_{\infty}^{2}\right)(2 l)}=\left(\frac{\sqrt{\mathrm{h}^{2}+1}+1}{\mathrm{~h}}\right)^{2 \beta} \mathrm{e}^{-\frac{2}{\pi \mathrm{h}}} \int_{-1}^{1} \frac{\tau(\mathrm{s})}{1+\mathrm{s}^{2} / \mathrm{h}^{2}} \mathrm{ds}
\end{aligned}
$$

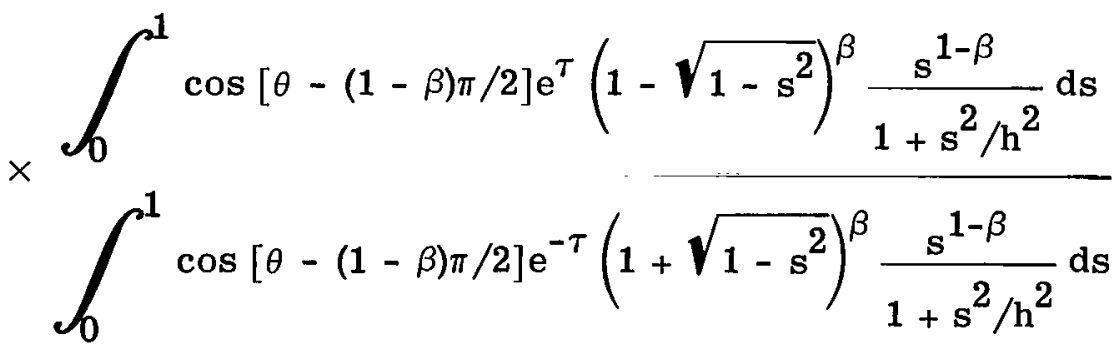

\section{NUMERICAL PROCEDURE}

Equations (30) and (40) were solved numerically with $\beta=2 / 3$ for various values of $\mathrm{h}$ (including $\mathrm{h}=\infty$ ) and with $\beta=1$ for $\mathrm{h}=\infty$. These equations were solved by iteration. First, $\theta$ was guessed as $\theta(\mathrm{s}) \propto \mathrm{s}$ and substituted into equation (40) to calculate $\tau(\mathrm{s})$. This $\tau(\mathrm{s})$ was used in equation (30) to calculate a new $\theta(\mathrm{s})$ which was then used in equation $(40)$ to calculate new values of $\tau(\mathbf{s})$. The procedure was repeated until convergence was obtained. In the application of this iteration procedure, certain special techniques had to be used. First, the numerical integration scheme used with equation (30) had to be tailored to take the principal value of the integral; that is, it had to be set up so that the singularities on both sides of the point $s^{\prime}=s$ cancelled one another in the principalvalue sense. Second, if any small bumps developed in the curve $\tau$ against $s$ during the course of the iteration process, it was necessary to smooth these out before the values of $\tau$ were substituted into equation (30) to calculate new values of $\theta$. This smoothing out 
was done because the singularity in equation (30) would tend to make these small bumps grow and thus lead to an unstable iteration process. Finally, it was necessary to use an averaged iteration procedure with respect to a weight factor $\epsilon$ (ref. 4, p. 216); that is, instead of equation (30) being used directly in the iteration processes, it was necessary to rewrite it in the form

$$
\theta(\mathbf{s})=\epsilon \theta(\mathrm{s})+(1-\epsilon) P . V \cdot \frac{1}{\pi} \int_{0}^{1} \frac{\tau\left(\mathbf{s}^{\prime}\right)}{\mathbf{s}^{\prime}-\mathbf{s}} \mathrm{d} \mathbf{s}^{\prime}
$$

so that, if $\theta^{(\mathrm{n})}$ and $\tau^{(\mathrm{n})}$ are the values of $\theta$ and $\tau$ obtained in the $\mathrm{n}^{\text {th }}$ step of the iteration process, the $n+1$ step value of $\theta$ is obtained from

$$
\theta^{(\mathrm{n}+1)}(\mathrm{s})=\epsilon \theta^{(\mathrm{n})}(\mathrm{s})+(1-\epsilon) \text { P. V. } \frac{1}{\pi} \int_{-1}^{1} \frac{\tau^{(\mathrm{n})}\left(\mathrm{s}^{\prime}\right)}{\mathrm{s}^{\prime}-\mathrm{s}}
$$

When these techniques were used, the iteration process converged to three significant figures in less than 10 iterations with $\epsilon=0.5$.

\section{RESULTS AND DISCUSSION}

The analysis shows that there are two possible solutions to the problem of determining the shape of an arc with the model used for the internal structure. One of these arcs has a sharp leading edge (figs. 6 and 7) with a $120^{\circ}$ included angle, and the other has a blunt leading edge (fig. 8). The solution for the arc with the sharp leading edge (with the channel walls separated by six or more arc widths, which corresponds to the experimental conditions) predicts an arc width-thickness (in the direction of flow) ratio of about 2 and a value of the experimentally observed parameter $s \mathrm{~B} /\left(\rho \mathrm{V}_{\infty}^{2} / 2\right)(2 l)$ between 0.6 and 0.8 (fig. 9). These results are in agreement with the experimental results of Roman and Myers (except those results at the lower velocities) (ref. 1) and the results of Myers (ref. 7). The dimensionless parameters shown in figure 9 are uniquely related to the distance $\mathrm{h}$ in the $t$-plane (fig. 4) according to the relations listed in the section Summary of Calculated Quantities. In particular, the parameter $h$ determines the shape of the arc. Even for an infinite stream $(h=\infty)$, a change in the stream velocity alone, for example, does not alter the shape of the arc because the magnetic field or current must be adjusted to maintain stability.

The arc with the blunt leading edge $(\beta=1)$ had too large a width-thickness ratio to 


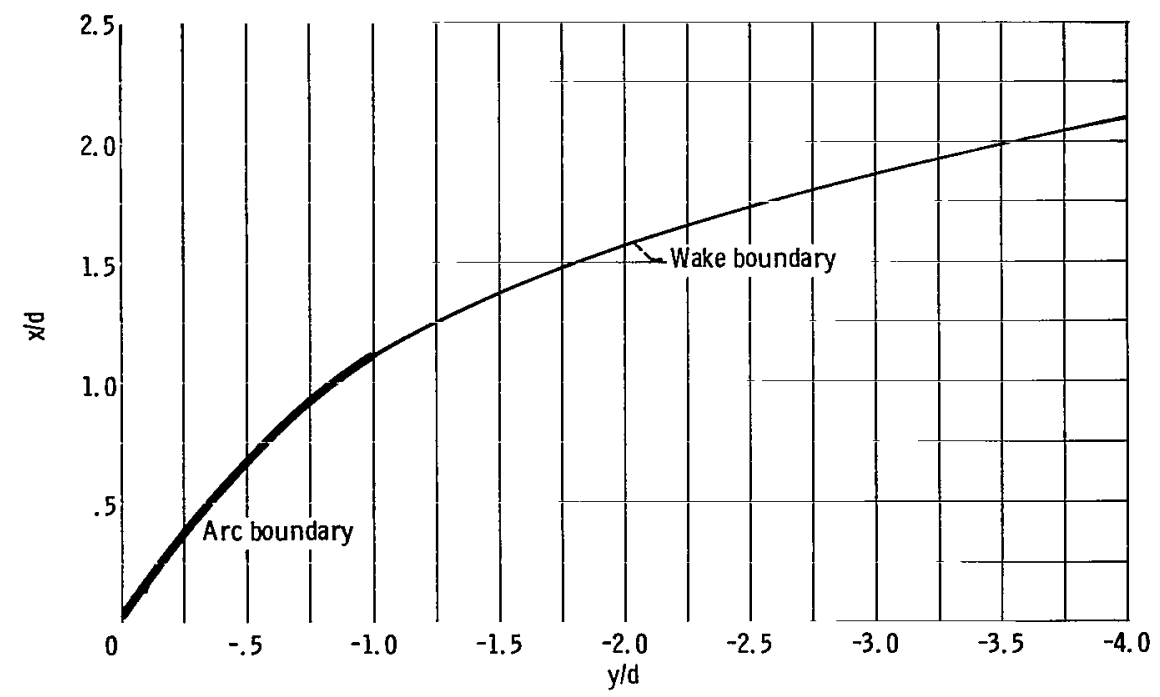

Figure 6. - Shape of arc with sharp leading edge in infinite stream. Distance between stagnation point and point at upstream infinity in t-plane, $\infty$; channel width, $\infty$; included angle between two tangents to arc surface at stagnation point divided by $\pi$. $2 / 3$.

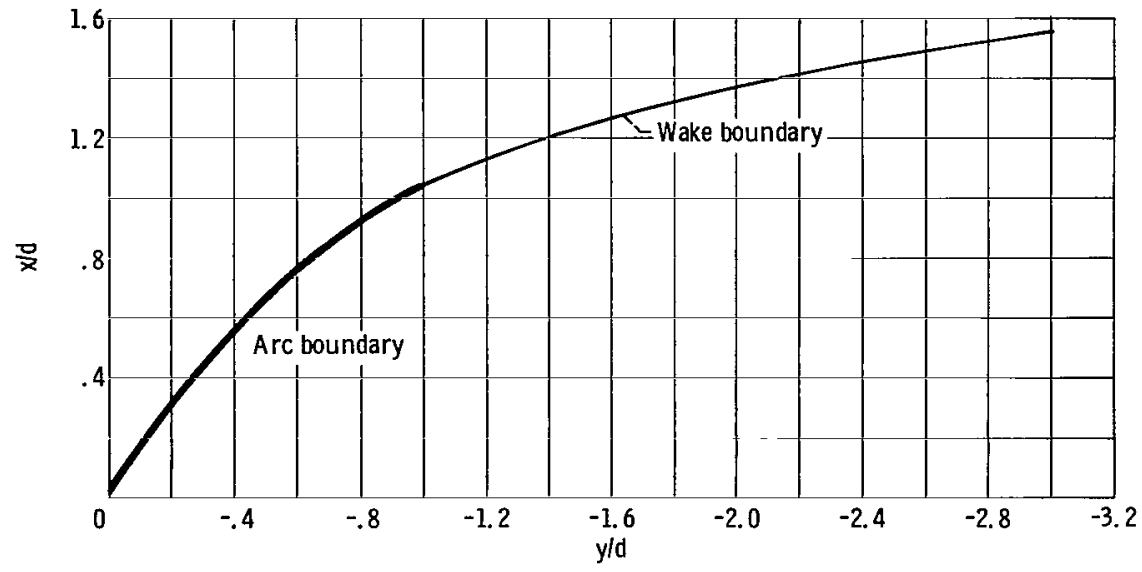

Figure 7. - Shape of arc and wake with sharp leading edge. Distance between stagnation point and point at upstream infinity in t-plane, 1; channel width, 5.88; included angle between two tangents to arc surface at stagnation point divided by $\pi, 2 / 3$. 


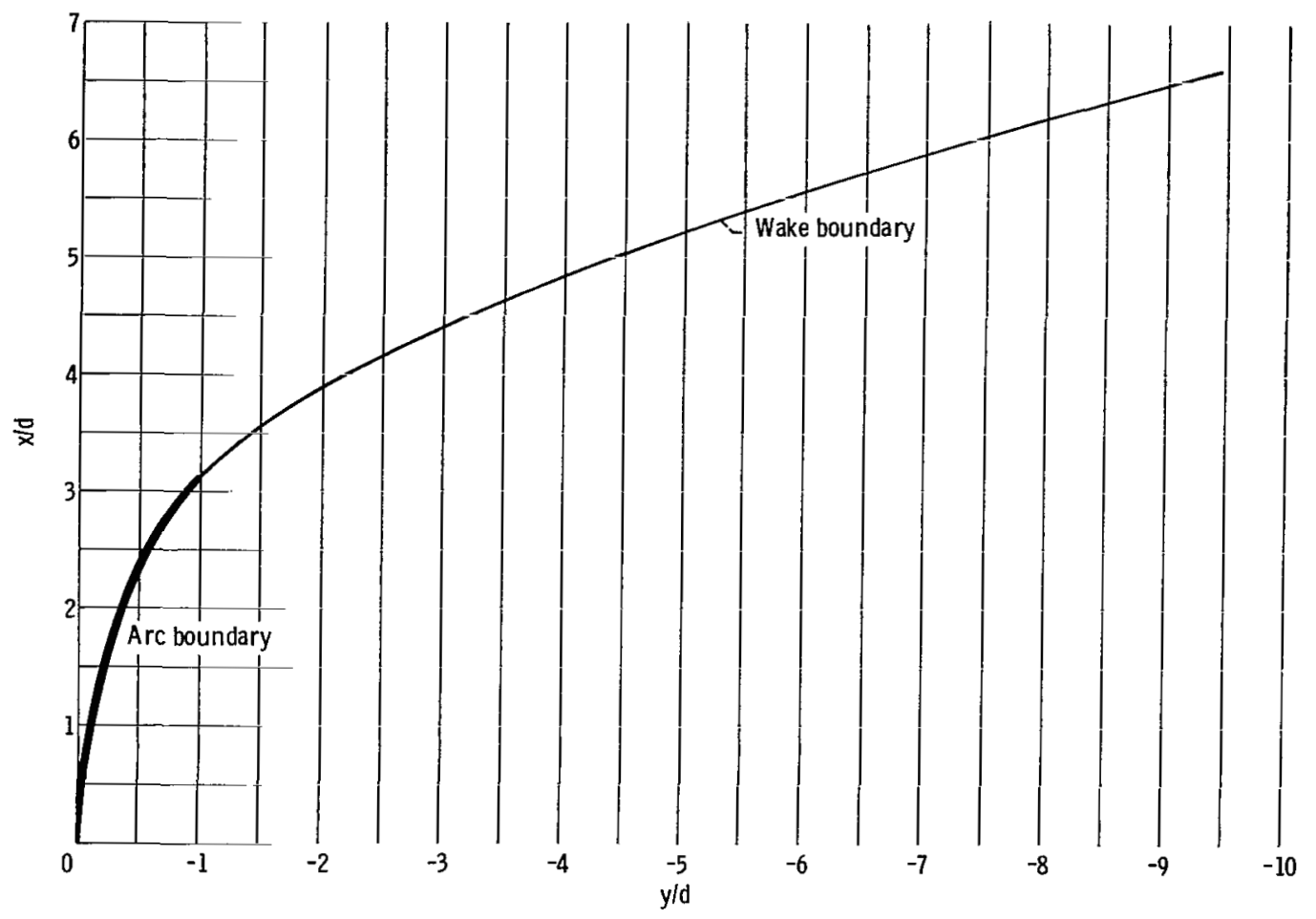

Figure 8. - Shape of arc with blunt leading edge in infinite stream. Distance between stagnation point and point at upstream infinity in t-plane, $\infty$; channel width, $\infty$; included angle between two tangents to arc surface at stagnation point divided by $\pi, 1$.

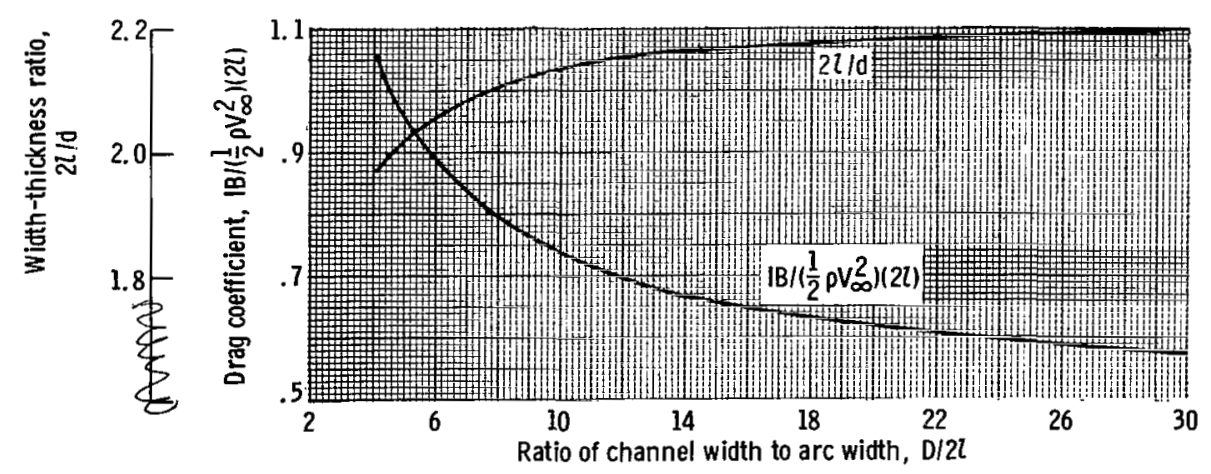

Figure 9. - Width-thickness ratio and drag coefficient for arc with sharp leading edge as function of ratio of channel width to arc width. Included angle between two tangents to arc surface at stagnation point divided by $\pi, 2 / 3$. 


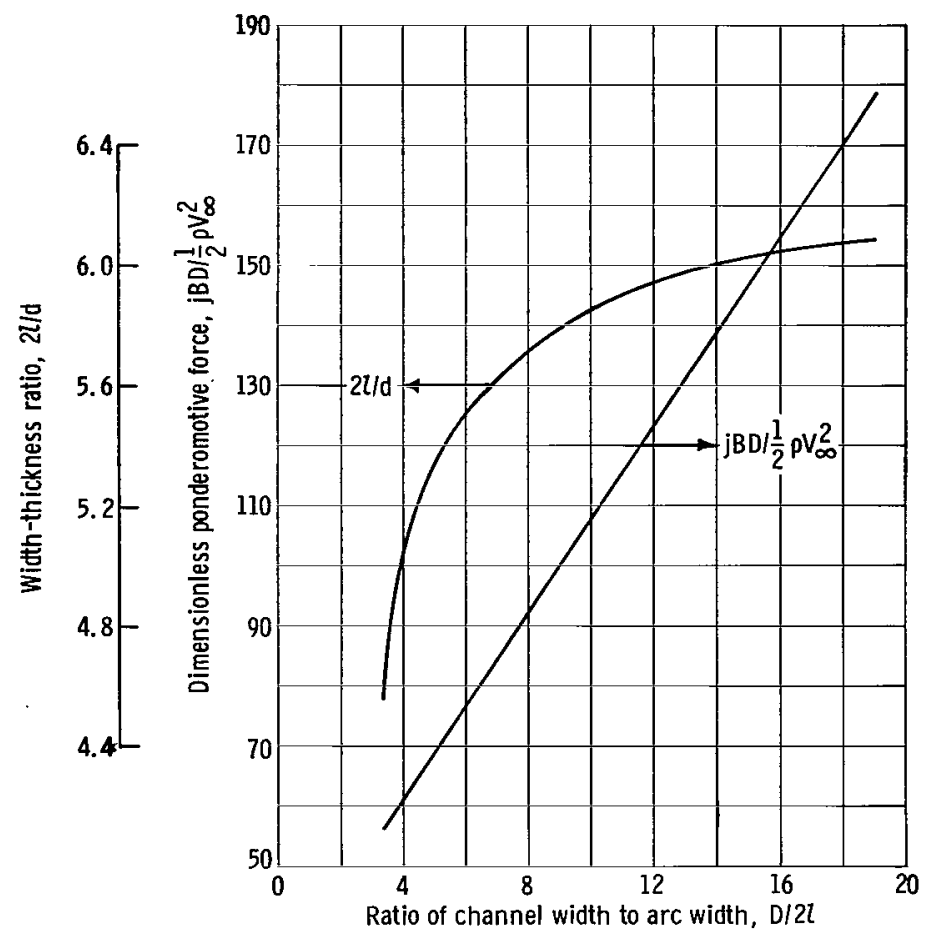

Figure 10. - Width-thickness ratio and dimensionless ponderomotive force as function of channel size for arc with blunt leading edge. Included angle between two tangents to arc surface at stagnation point divided by $\pi, 1$.

agree with the experiments. The geometric and dynamic parameters for this type of arc are shown in figure 10. The actual bluntness observed in experiments must be attributed to transport effects such as viscosity. The model also predicts the broad wake region observed experimentally. At this stage, it is not as yet possible to make any more comparisons with the experimental results.

The techniques developed herein can be used easily with more general models of the internal structure of the arc to obtain more accurate results.

\section{CONCLUSION}

A previous analysis of the shape of a magnetically balanced arc was improved by giving a more accurate description of the external flow field. Two possible solutions were obtained for the model used. One solution was in good agreement with experimental results whereas the other was not. The model used for the internal structure of the arc is expected to be most accurate at the higher flow velocities. 
The techniques developed in this report can be used easily with more general models of the internal structure of the arc to obtain more accurate results.

Lewis Research Center,

National Aeronautics and Space Administration, Cleveland, Ohio, May 31, 1968, 129-01-11-05-22.

\section{REFERENCES}

1. Roman, W. C.; and Myers, T. W. : Experimental Investigation of an Electric Arc in Transverse Aerodynamic and Magnetic Fields. AIAA J., vol. 5, no. 11, Nov. 1967, pp. 2011-2017.

2. Goldstein, M. E.; and Fay, J. A.: The Shape of a Magnetically Balanced Arc. AIAA J., vol. 5, no. 8, Aug. 1967, pp. 1510-1511.

3. Churchill, Ruel V.: Complex Variables and Applications. Second ed., McGraw-Hill Book Co., Inc., 1966.

4. Birkhoff, Garrett; and Zarantonello, E. H.: Jets, Wakes, and Cavities. Academic Press, Inc., 1957.

5. Rudin, Walter: Real and Complex Analysis. McGraw-Hill Book Co., Inc., 1966.

6. Gakhov, F. D. : Boundary Value Problems. Second rev., Addition-Wesley Publ. Co., Inc., 1966.

7. Myers, T. W. : Experimental Investigation of the Balancing Mechanism and Electrical Properties of an Argon Arc in Crossed Convective and Magnetic Fields. Presented at the Seventh Symposium on Engineering Aspects of Magnetohydrodynamics, Princeton, 1966. 


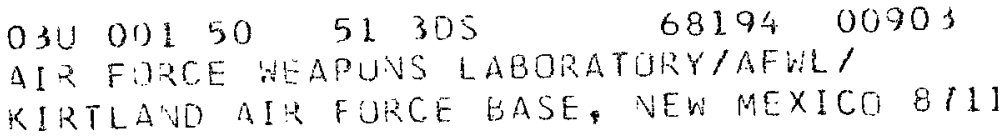

AYT MISS MADELIHF F CAMUVA CHIEF TFEH LIBRAKY/WIL/

POSTMASTER:

"The aeronautical and space activities of the United States shall be conducted so as to contribute. . . to the expansion of buman knowledge of phenomena in the atmosphere and space. The Administration shall provide for the widest practicable and appropriate dissemination of information concerning its activities and the results thereof."

- National Aeronautics ANd Space ACt of 1958

\section{NASA SCIENTIFIC AND TECHNICAL PUBLICATIONS}

TECHNICAL REPORTS:: Scientific and. technical information considered important, complete, and a lasting contribution to existing knowledge.

TECHNICAL NOTES: Information less broad in scope but nevertheless of importance as a contribution to existing knowledge.

TECHNICAL MEMORANDUMS:

Information receiving limited distribution because of preliminary data, security classification, or other reasons.

CONTRACTOR REPORTS: Scientific and technical information generated under a NASA contract or grant and considered an important contribution to existing knowledge.
TECHNICAL TRANSLATIONS: Information published in a foreign language considered to merit NASA distribution in English.

SPECIAL PUBLICATIONS: Information derived from or of value to NASA activities. Publications include conference proceedings, monographs, data compilations, handbooks, sourcebooks, and special bibliographies.

TECHNOLOGY UTILIZATION PUBLICATIONS: Information on technology used by NASA that may be of particular interest in commercial and other non-aerospace applications. Publications include Tech Briefs, Technology Utilization Reports and Notes, and Technology Surveys.

Defails on the availability of these publications may be obtained from:

SCIENTIFIC AND TECHNICAL INFORMATION DIVISION

NATIONAL AERONAUTICS AND SPACE ADMINISTRATION

Washington, D.C. 20546 